\title{
Extrapulmonary tuberculosis in the setting of HIV hyperendemicity at a tertiary hospital in Durban, South Africa
}

\author{
S Gounden ${ }^{a *}$ iD R Perumal $^{b}$ and NP Magula ${ }^{a}$ iD \\ aDepartment of Internal Medicine, School of Clinical Medicine, University of KwaZulu-Natal, Durban, South Africa \\ ${ }^{b}$ Department of Pulmonology and Critical Care, Centre for the AIDS Programme of Research in South Africa, University of KwaZulu-Natal, Durban, \\ South Africa \\ *Corresponding author, email: vaseng23@gmail.com

Background: South Africa has the highest burden of tuberculosis/human immunodeficiency virus (TB/HIV) co-infection in the world, with the province of KwaZulu-Natal representing the global epicentre of TB/HIV. While significant progress has been made to improve the diagnosis of pulmonary tuberculosis, the diagnosis of extrapulmonary TB (EPTB) remains a significant challenge in resource-constrained settings.

Methods: A retrospective chart review was conducted, and included all adult patients diagnosed with EPTB at a tertiary hospital in Durban, South Africa, between January 1, 2016 and March 31, 2016.

Results: There were 188 new cases of TB during the study period, with 80 patients diagnosed with EPTB. The mean age of patients was 34.73 (SD \pm 9.44 ) years. Forty-two $(52.5 \%)$ patients were female, while $76(96 \%)$ were black. The most common risk factor for EPTB was HIV co-infection (88.8\%). The median CD4 cell count was 68 (IQR 32-165) cells/mm³. Pleural (36.3\%), lymph node (28.7\%) and abdominal (27.5\%) involvement were the most common sites of disease Weight loss, fever, night sweats and cough were amongst the most common symptoms reported. A microbiologically confirmed diagnosis was made in only $65 \%$ of cases. In the majority of cases, more than one diagnostic method was used to confirm the presence of TB in distant organs.

Conclusion: Immunosuppression, most commonly by HIV, remains the most significant risk factor for the development of EPTB. Advancements in Xpert MTB/Rif and computed tomography have assisted in increasing the diagnostic armamentarium of EPTB. Despite improved access to antiretroviral therapy over the past years, advanced HIV disease remains a significant challenge to TB control.

Keywords: HIV, extrapulmonary TB, risk factors for extrapulmonary TB, signs and symptoms of extrapulmonary TB

Tuberculosis (TB) describes many clinical illnesses caused by Mycobacterium tuberculosis.' Globally in 2013, nine million people developed TB, with 1.5 million tuberculosis-related deaths reported in the same period. In the same year, there were 328826 cases of TB reported in South Africa, 37709 cases of which were extrapulmonary $\mathrm{TB}(\mathrm{EPTB}) .^{2}$ Tuberculosis remains one of the leading causes of death in the country. ${ }^{3}$ KwaZulu-Natal has the highest prevalence of TB in the country with 1076 notified cases per 100000 population. ${ }^{4}$

Tuberculosis is the most common human immunodeficiency virus (HIV) associated disease, and represents the leading cause of death in these patients globally. ${ }^{5}$ The presentation of the disease may be related to the level of immunosuppression of the patient. Extrapulmonary involvement in particular has been associated with advanced HIV infection..$^{6-8}$ Overall, pulmonary TB is more common than EPTB in people living with HIV. ${ }^{6}$ After more than a decade of rolling out the largest antiretroviral programme in the world, more than 2.4 million people now have access to ART in South Africa. ${ }^{9}$

Many risk factors for development of EPTB have been identified. One of the key risk factors is a compromised host immunity, particularly a weak cellular immunity. ${ }^{10}$ Diabetes, smoking, malignancy, low body weight, chronic kidney disease and the use of immunosuppressive drugs represent secondary causes of immunosuppression and have also been associated with an increased risk of developing EPTB. ${ }^{11}$ Diabetic patients with EPTB had an increased risk of death when compared with non-diabetic patients with EPTB. ${ }^{12}$ Classically, young children and the elderly carried a disproportionately increased risk of EPTB. ${ }^{13}$ However, the high burden of HIV in the young adult population in our setting has seen an increased burden of tuberculosis in this group. ${ }^{14}$ Low socio-economic status has been shown to confer an increased risk for EPTB. ${ }^{8}$

EPTB, especially invasive disease, presents more commonly in patients with HIV co-infection. In patients with advanced HIV with CD4 cell counts of less than 250 cells $/ \mathrm{mm}^{3}$, disease progression is markedly different compared with patients with higher CD4 cell counts. ${ }^{15}$ Symptoms may be subacute or acute with rapid progression and death. ${ }^{7}$ The clinical manifestations are variable, but often reflect the organs involved..$^{15}$ Common sites of infection include the pleura, central nervous system, lymphatic system, genitourinary system and musculoskeletal system. ${ }^{8}$ The diagnosis of EPTB remains challenging due to the protean presentation of the disease and a high index of suspicion is required when assessing a patient with possible EPTB. ${ }^{16}$ Fever, weight loss, anorexia and malaise have been documented as the most common symptoms reported by patients with EPTB regardless of the site of disease, but this constitutional syndrome alone has a poor positive predictive value for the diagnosis of TB. ${ }^{15,17}$

EPTB poses a significant diagnostic dilemma. Due to the high variability in the clinical presentation of the disease, clinicians are reliant on a high index of suspicion and on laboratory and radiological tests to establish the diagnosis. A prospective cohort study in the high TB/HIV burden setting of South Africa demonstrated the utility of expanded case definitions for the 
diagnosis of EPTB in HIV-infected adults, and reported this to be an effective strategy in such individuals. ${ }^{17}$ Collectively, the suggested expanded case definitions for the diagnosis of EPTB had a positive predictive value of $88 \%$, and the study emphasised

Table 1: Sociodemographic characteristics, risk factors, and sites of EPTB
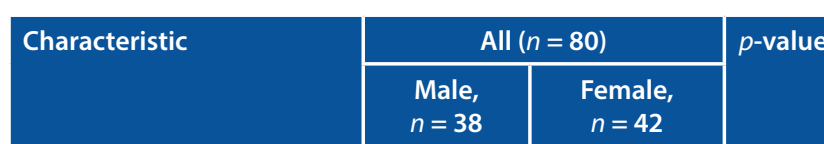

Age

Age categories:

$<30$ years

30 to 39

40 to 49

50 or older

Ethnicity:

African

Asian

Caucasian

Living economy:

Rural

Urban

Employment status/high-risk

environment:

Unemployed

Private sector

Correctional service

Healthcare facility

Presence of risk factors for TB (proportion of patients):

Past TB infection

HIV co-infection

CKD

Alcohol/smoking

Diabetes mellitus

Immunosuppressant

Low body weight

Head/neck malignancy

Mean CD4 cell count ${ }^{*}$

ART naive

Sites of EPTB:

Only 1 EPTB site

$>1$ EPTB site

Pulmonary

Lymph node

Pleura

Bone

Abdomen

CXR with miliary pattern

Pericardium

Meninges

Blood/bone marrow

Data expressed as $n$ (\%) or as mean \pm standard deviation; \# cells $/ \mathrm{mm}^{3}$.

$\mathrm{TB}=$ tuberculosis; $\mathrm{EPTB}=$ extra-pulmonary tuberculosis; $\mathrm{CKD}=$ chronic kidney disease; $\mathrm{ART}=$ antiretroviral therapy; $\mathrm{CXR}=$ chest $\mathrm{X}$-ray . the need for an objective evaluation of response to treatment when the diagnosis of EPTB is made without microbiological evidence. ${ }^{17}$ The gold standard for the diagnosis of tuberculosis remains culture of the organism. In EPTB, the long turn-around

\begin{tabular}{|c|c|c|c|c|c|c|c|}
\hline$n=38$ & $n=42$ & & $n=31$ & $n=40$ & & & \\
\hline $36.6 \pm 8.7$ & $33.1 \pm 9.9$ & 0.09 & $35.5 \pm 7.0$ & $33.3 \pm 10.0$ & 0.30 & $41.0 \pm 13.8$ & $28 \pm 5.6$ \\
\hline & & 0.12 & & & 0.13 & & \\
\hline $7(18.4)$ & $15(35.7)$ & & $6(19.4)$ & $14(35)$ & & $1(14.3)$ & $1(50)$ \\
\hline $19(50)$ & $20(47.6)$ & & $17(54.8)$ & $19(47.5)$ & & $2(28.6)$ & $1(50)$ \\
\hline $9(23.7)$ & $3(7.2)$ & & $7(22.6)$ & $3(7.5)$ & & $2(28.6)$ & $0(0)$ \\
\hline $3(7.9)$ & $4(9.5)$ & & $1(3.2)$ & $4(10)$ & & $2(28.6)$ & $0(0)$ \\
\hline 34 (89.5) & $42(100)$ & & $30(96.8)$ & $40(100)$ & & $4(57.1)$ & $2(100)$ \\
\hline $3(7.9)$ & $0(0)$ & & $1(3.2)$ & $0(0)$ & & $2(28.6)$ & $0(0)$ \\
\hline $1(2.6)$ & $0(0)$ & & $0(0)$ & $0(0)$ & & $1(14.3)$ & $0(0)$ \\
\hline & & 0.63 & & & 0.44 & & \\
\hline $5(13.2)$ & $4(9.5)$ & & $5(16.1)$ & $4(10)$ & & $0(0)$ & $0(0)$ \\
\hline $33(86.8)$ & $38(90.5)$ & & $26(83.9)$ & $36(90)$ & & $7(100)$ & $2(100)$ \\
\hline & & 0.58 & & & 0.61 & & \\
\hline $25(65.8)$ & $32(76.2)$ & & $21(67.7)$ & 31 (77.5) & & $4(57.1)$ & $1(50)$ \\
\hline $10(26.3)$ & 8 (19) & & $7(22.6)$ & 7 (17.5) & & $3(42.9)$ & $1(50)$ \\
\hline $3(7.9)$ & $1(2.4)$ & & $3(9.7)$ & $1(2.5)$ & & $0(0)$ & $0(0)$ \\
\hline $0(0)$ & $1(2.4)$ & & $0(0)$ & $1(2.5)$ & & $0(0)$ & $0(0)$ \\
\hline $8(21.1)$ & $8(19)$ & 0.81 & $8(25.8)$ & $8(20)$ & 0.57 & $0(0)$ & $0(0)$ \\
\hline 31 (81.6) & $40(95.2)$ & 0.06 & $31(100)$ & $40(100)$ & N/A & - & - \\
\hline $3(7.9)$ & $4(9.5)$ & 0.80 & $1(3.2)$ & $4(10)$ & 0.27 & $2(28.6)$ & $0(0)$ \\
\hline $24(63.2)$ & $12(28.6)$ & $<0.05$ & $19(61.3)$ & $11(27.5)$ & $<0.05$ & $5(71.4)$ & $1(100)$ \\
\hline $4(10.5)$ & $1(2.4)$ & 0.14 & $1(3.2)$ & $1(2.5)$ & 0.86 & $3(42.9)$ & $0(0)$ \\
\hline $2(5.3)$ & $1(2.4)$ & 0.50 & $2(6.5)$ & $1(2.5)$ & 0.41 & $0(0)$ & $0(0)$ \\
\hline 15 (39.5) & $15(35.7)$ & 0.73 & $14(45.2)$ & $15(37.5)$ & 0.52 & $1(14.3)$ & $0(0)$ \\
\hline $1(2.6)$ & $2(4.8)$ & 0.61 & $0(0)$ & $2(5.0)$ & 0.21 & $1(14.3)$ & $0(0)$ \\
\hline 102.9 (11.6) & $120.7(13.8)$ & $<0.05$ & $102.9(11.6)$ & $120.7(13.8)$ & $<0.05$ & - & - \\
\hline $15(48.4)$ & $22(55)$ & 0.58 & $15(48.4)$ & $22(55)$ & 0.58 & - & - \\
\hline 30 (78.9) & 35 (83.3) & & $24(77.4)$ & $33(82.5)$ & & $6(85.7)$ & $2(100)$ \\
\hline $8(21.1)$ & 7 (16.7) & & 7 (22.6) & 7 (17.5) & & $1(14.3)$ & $0(0)$ \\
\hline $3(7.9)$ & $2(4.8)$ & & $2(6.5)$ & $2(5.0)$ & & $1(14.3)$ & $0(0)$ \\
\hline 12 (31.6) & $11(26.2)$ & & $12(38.7)$ & $11(27.5)$ & & $0(0)$ & $0(0)$ \\
\hline $13(34.2)$ & $16(38.1)$ & & $9(29)$ & $16(40)$ & & $4(57.1)$ & $0(0)$ \\
\hline $1(2.6)$ & $1(2.4)$ & & $0(0)$ & $1(2.5)$ & & $1(14.3)$ & $0(0)$ \\
\hline $11(28.9)$ & $11(26.2)$ & & $9(29)$ & $9(22.5)$ & & $2(28.6)$ & $2(100)$ \\
\hline $0(0)$ & $2(4.8)$ & & $0(0)$ & $2(5.0)$ & & $0(0)$ & $0(0)$ \\
\hline $3(7.9)$ & $1(2.4)$ & & $3(9.7)$ & $1(2.5)$ & & $0(0)$ & $0(0)$ \\
\hline $4(10.5)$ & $4(9.5)$ & & 4 (12.9) & $4(10)$ & & $0(0)$ & $0(0)$ \\
\hline $1(2.6)$ & $1(2.4)$ & & $1(3.2)$ & $1(2.5)$ & & $0(0)$ & $0(0)$ \\
\hline
\end{tabular}


time associated with culture creates an unacceptable delay in diagnosis and treatment, and contributes to the increased mortality in this group. Liquid culture medium, however, has a shorter turn-around time compared with conventional solid medium. ${ }^{18}$ In a resource-limited environment like South Africa, the need for expensive and sophisticated laboratory equipment presents another challenge to the routine use of culture.

During the last decade, scientific advancements have assisted in improving diagnosis of EPTB. The increasing use of adenosine deaminase (ADA) levels and nucleic acid amplification techniques like Xpert MDR/Rif on body fluid and tissue samples have improved our diagnostic armamentarium. These tests have assisted in improving the diagnosis of EPTB in sputum, urine, blood and cerebrospinal fluid. ${ }^{15,19}$ Xpert MTB/Rif has a high specificity regardless of the tissue sampled; however, sensitivity varies significantly. ${ }^{20}$ Compromised sensitivity limits the role of this test in ruling out disease. In a recent study by Naidoo et al. the introduction of Xpert MDR/Rif in the South African context has substantially increased the cost of diagnosis of pulmonary tuberculosis (PTB), without the expected increase in diagnostic efficiency that was predicted by an earlier population-level decision model..$^{21,22}$ Importantly, no similar studies exist for evaluating the costeffectiveness of Xpert MDR/Rif in the context of EPTB. Nonetheless, the use of Xpert MDR/Rif can be used by non-laboratory technicians in decentralised models, thereby reducing the costs associated with transportation of specimens and theoretically reducing diagnostic delay. ${ }^{23}$ Cox et al. found that in Khayelitsha the use of decentralised Xpert MDR/Rif led to the improved case detection of rifampicin-resistant TB and a significantly reduced time to treatment of drug-resistant TB. ${ }^{24}$ The role of Xpert MTB/Rif in diagnosing TB in specimens other than sputum has revealed its potential utility in diagnosing EPTB. Xpert MTB/Rif has a sensitivity between $77.6 \%$ and $100 \%$ depending on the specimen involved, while the specificity ranges between 73.3 and $98.3 \% .^{20,25,26}$

ADA is an enzyme that catalyses the conversion of adenosine to inosine and plays an important role in the differentiation of lymphoid cells. Diseases that stimulate cellular immunity increase ADA levels. ${ }^{27}$ Lymphocyte-predominant pleural effusions with elevated ADA suggest pleural TB or malignancies. ${ }^{27}$ ADA levels over $40 \mathrm{U} / \mathrm{I}$ with more than $50 \%$ lymphocyte proportion suggest pleural TB (sensitivity $90.7 \%$ [Cl $87.3-94.1 \%]$; specificity $97.7 \%$ [Cl 95.9-99.5\%]). ${ }^{28}$ Although pleural biopsy is the most effective method of diagnosis of TB pleural effusions, ADA remains a reliable and inexpensive tool in a high-prevalence setting. ${ }^{29}$ In a recent meta-analysis of the role of ADA in the diagnosis of tuberculous pleural effusions, ADA was demonstrated to have good diagnostic accuracy, and has been suggested as a rule-out test in resource-constrained settings. ${ }^{30,31}$ False-positive results are a concern, but this is confounded by the low yield of positive TB culture on pleural fluid, which is often used as the gold standard when evaluating the performance of this test. ${ }^{32}$ Suggested cutoff values for ADA are as follows: ascitic fluid $>39 \mathrm{U} / \mathrm{l}$, cerebrospinal fluid $>6 \mathrm{U} / \mathrm{l}$ and pleural fluid $>40 \mathrm{U} / \mathrm{I}^{31,33,34}$

The urine-based, lateral flow, point-of-care, lipoarabinomannan assay (LAM) has been demonstrated to have a sensitivity of $57.7 \%(95 \% \mathrm{Cl} 51.0-64.2)$ and specificity of $85.5 \%$ (95\% Cl $81.8-$ 88.6) for the diagnosis of tuberculosis in hospitalised patients with advanced immunosuppression (CD4 cell count $<100$ cells per $\mu \mathrm{ll}){ }^{35}$ In this subset of patients, LAM-guided, prompt antituberculosis treatment initiation reduced mortality by up to $29 \% .{ }^{35}$ However, the test performed poorly and had no incremental yield over Xpert MTB/Rif or sputum microscopy in
HIV-infected outpatients, even when advanced immunosuppression was present. ${ }^{36}$ Nonetheless, the test remains an important potential tool for the prompt diagnosis of EPTB in hospitalised, HIV-infected individuals with advanced immunosuppression who are most at risk of death.

Computed tomography (CT) scanning has been shown to significantly increase the detection of abdominal TB in inconclusive cases but its utility is limited by the scarcity of CT facilities in our setting. ${ }^{37}$ Abdominal ultrasound is a useful tool in the diagnosis of abdominal tuberculosis and is the most widely used screening study for suspected abdominal involvement. A variety of ultrasonographic features may suggest active TB, most commonly the presence of abdominal lymphadenopathy. ${ }^{38}$ Patel et al. used ultrasonography to assess abdominal involvement in patients with pulmonary TB at a South African hospital and found that abdominal lymphadenopathy over $1 \mathrm{~cm}$, splenic lesions with lymphadenopathy, and/or ascites, were significant findings $(p<0.05) .^{39}$

Due to the variability of presentation of EPTB, a standardised diagnostic algorithm remains elusive. A high index of suspicion in the appropriate clinical setting is necessary to direct further investigation. The study aims to describe the profile of patients with EPTB diagnosed at a tertiary facility in a setting of TB/HIV hyperendemicity.

\section{Methods}

A retrospective chart review was conducted of all adult patients diagnosed with EPTB at a tertiary hospital in Durban, KwaZuluNatal between January 1, 2016 and March 31, 2016. All adult cases were identified from the facility TB register, and clinical data were extracted from the associated clinical records. EPTB was defined as the involvement of any organ system outside the

Table 2: Distribution of sites of EPTB in HIV co-infected individuals ( $\boldsymbol{n}=71$ ) according to $\mathrm{CD}_{4}$ cell count and sex

\begin{tabular}{|c|c|c|c|c|}
\hline \multirow[t]{2}{*}{$\begin{array}{l}\text { Characteristic } \\
n(\%)\end{array}$} & \multicolumn{2}{|c|}{$\begin{array}{l}C_{4} \text { cell count less } \\
\text { than } 100 \text { cells } / \mathrm{mm}^{3}\end{array}$} & \multicolumn{2}{|c|}{$\begin{array}{l}C_{4} \text { cell count more than } \\
100 \text { cells } / \mathrm{mm}^{3}\end{array}$} \\
\hline & $\begin{array}{l}\text { Male, } \\
n=21\end{array}$ & $\begin{array}{c}\text { Female, } \\
n=25\end{array}$ & $\begin{array}{l}\text { Male, } \\
n=10\end{array}$ & $\begin{array}{c}\text { Female, } \\
n=15\end{array}$ \\
\hline Age & $35.5 \pm 6.2$ & $35.4 \pm 11.5$ & $35.5 \pm 8.7$ & $29.9 \pm 5.8$ \\
\hline $\begin{array}{l}\text { Only } 1 \text { extrapul- } \\
\text { monary site }\end{array}$ & $15(71.4)$ & $19(76.0)$ & $9(90.0)$ & $14(93.3)$ \\
\hline $\begin{array}{l}>1 \text { extrapulmo- } \\
\text { nary site }\end{array}$ & $6(28.6)^{*}$ & $6(24.0)^{*}$ & $1(10.0)$ & $1(6.7)$ \\
\hline Pulmonary** & $1(4.8)$ & $2(8.0)$ & $1(10.0)$ & $0(0.0)$ \\
\hline Lymph node & $10(47.6)$ & $6(24.0)$ & $2(20.0)$ & $5(33.3)$ \\
\hline Pleura & $5(23.8)$ & $10(40.0)$ & $4(40.0)$ & $6(40.0)$ \\
\hline Bone & $0(0)$ & $0(0.0)$ & $0(0.0)$ & $1(6.7)$ \\
\hline Abdomen & $8(38.1)$ & $7(28.0)$ & $1(10.0)$ & $2(13.3)$ \\
\hline $\begin{array}{l}\text { CXR with miliary } \\
\text { pattern }\end{array}$ & $0(0)$ & $0(0)$ & $0(0.0)$ & $2(13.3)$ \\
\hline Pericardial & $1(4.8)$ & $1(4.0)$ & $2(20.0)$ & $0(0.0)$ \\
\hline Meninges & $3(14.3)$ & $4(16.0)$ & $1(10.0)$ & $0(0.0)$ \\
\hline $\begin{array}{l}\text { Blood or bone } \\
\text { marrow }\end{array}$ & $0(0)$ & $1(4.0)$ & $0(0.0)$ & $0(0.0)$ \\
\hline
\end{tabular}

Data expressed as $n$ (\%) or as mean \pm standard deviation.

EPTB = extrapulmonary tuberculosis, $\mathrm{CXR}=$ chest radiograph

Statistically significant results indicated in bold.

*Indicates $p<0.05$ (comparing individuals of the same sex with CD4 cell counts greater than 100 cell $/ \mathrm{mm}^{3}$ ). Independent sample Student's t-test.

**Patients had confirmed pulmonary TB and another site of TB infection. 
lung parenchyma. Patients who had both pulmonary and EPTB were classified as EPTB. All data was analysed using SPSS ${ }^{\circledR} 23.0$ software (IBM Corp, Armonk, NY, USA). For all statistical comparisons, a 5\% level of significance was used; correspondingly $95 \%$ confidence intervals were used to describe effect size. All data were assessed for normality, and non-parametric tests were used where necessary. Medians and inter-quartile ranges were used for data not amenable to parametric description. Pearson's chi-square test or Fisher's exact test was used to assess the association between categorical variables of interest.

The study was conducted under the oversight of the University of KwaZulu-Natal Biomedical Research Ethics Committee (BREC no: BE283/16). The necessary permissions were obtained from health-system gatekeepers.

\section{Results}

During the period under review, 188 new cases of TB were notified, and included 80 cases of EPTB (Table 1). The mean age of patients was $34.73(\mathrm{SD} \pm 9.44)$ years. Forty-two $(52.5 \%)$ patients were female, and the majority (96\%) of patients were black. Patients were predominantly from urban areas. Fifty-seven (71.2\%) patients were unemployed, while five patients were employed in high-risk working environments (one in a health facility, and four in correctional services facilities). The most common risk factor for EPTB was HIV co-infection (88.8\%). The mean CD4 cell count was 102.9 (SD 11.6) cells per $\mu$ in men and 120.7 (SD 13.8) cells per $\mu$ in women. Less than half (42.5\%) of the patients were on ART prior to the diagnosis of EPTB. Tobacco use was reported in 44 (55\%) patients. Smoking and alcohol use was more common in males $(63.2 \%$ vs. $28.6 \%, p<0.05)$. Chronic kidney disease, diabetes mellitus, malignancy and immunosuppressive therapy contributed minimally to the overall risk factors for EPTB.

Pleural (36.3\%), lymph node (28.8\%) and abdominal (27.5\%) involvement were the most common. Fifteen (18.8\%) patients had tuberculosis diagnosed at more than one anatomical site. Although the sites of involvement between males and females were fairly evenly distributed in the HIV-infected population, the presence of pleural involvement in females (40\%) was more frequent than that in males (29\%). In the HIV-uninfected/ unknown population, abdominal involvement was the only site that was involved in females.

In the subgroup of patients who had pulmonary and extrapulmonary disease, four of the five patients were infected with HIV. Abdominal involvement was the most common in this subgroup (three of the five patients). The remaining two patients had pericardial and pleural involvement respectively.

There were no statistically significant differences between patients with CD4 cell counts of less than 100 cells $/ \mathrm{mm}^{3}$ and those patients with CD4 cell counts of more than 100 cells $/ \mathrm{mm}^{3}$ except that both males and females in the lower CD4 cell count subgroup presented more frequently with EPTB involving more than one site $(p<0.05)$ compared with patients with CD4 cell counts of more than 100 cells $/ \mathrm{mm}^{3}$ (Table 2).

On analysis of symptoms with which patients presented, constitutional symptoms were the most frequent (Table 3). Unintentional weight loss and fever were the predominant symptoms regardless of site of disease. Night sweats were present in almost half of the cases regardless of the system affected, except for the patients with bone involvement.
Although weight loss and fever were the most frequent complaints, these was not associated with any specific disease site. Only a few symptoms were specific for the system affected. Lymph node enlargement was only reported with TB lymphadenitis, as were joint symptoms limited to bone involvement. Respiratory symptoms like cough, dyspnoea and chest pain were predominant in pulmonary and pleural involvement but also in TB of other sites except bone involvement. Headache, photophobia and confusion were significant symptoms in TB meningitis $(p<0.05)$. In patients with abdominal involvement, weight loss was significantly more frequent $(p<0.05)$. As expected, other gastrointestinal symptoms like abdominal pain, diarrhoea and vomiting were more common in patients with abdominal TB $(p<0.05)$. Clinical signs showed a greater degree of association with the site of EPTB (Table 4). Significantly, all cases of TB involving the lymphatic system had lymphadenopathy. Similarly, all cases of meningeal involvement had meningism. Peritonitis, ascites and hepatomegaly were significantly more common in cases of abdominal tuberculosis.

A microbiologically proven diagnosis was made in only 52 (65\%) cases of EPTB. In 53 (66.25\%) cases the diagnosis was made using a combination of microbiological, radiological and chemistry data (Table 5). Cerebrospinal fluid and pleural fluid were sent for Xpert MTB/Rif assessment. A positive Xpert result supported the diagnosis of TB meningitis in five $(62.5 \%)$ out of the eight cases. In cases of pleural tuberculosis, Xpert MTB/Rif supported the diagnosis in one (3.8\%) of 27 cases. The diagnosis of TB pleura was supported by the presence of an exudative pleural effusion with an elevated pleural fluid ADA in most cases. The median total protein on pleural fluid assessment was $57 \mathrm{mmol} / \mathrm{l}$ (IQR 52-62 mmol/I), median LDH $985 \mathrm{mmol} / \mathrm{I}$ (IQR 637-1 $252 \mathrm{mmol} / \mathrm{I})$ and median ADA was $45 \mathrm{U} / \mathrm{L}$ (IQR 40-57 U/I).

\section{Discussion}

This study provides a description of patients with EPTB in a high TB/HIV burden setting more than a decade into universal access to ART. Patients with EPTB in this setting were predominantly young $(34.73 \pm 9.44)$ years and female $(52.5 \%)$. This is different from settings of low HIV prevalence, where TB continues to affect predominantly children and the elderly. The increased burden of TB among young adults is consistent with the findings in other high HIV burden settings, where HIV has been described as the key driver of TB. ${ }^{14,40}$ Similarly, the significant representation of women in this group of patients with EPTB is likely a result of the high HIV prevalence in young women in our setting. ${ }^{41,42}$ Studies in Turkey and Brazil have confirmed similar prevalence amongst women, as a result of their disproportionate burden of HIV in young women resulting from a range of biological and systematic factors. ${ }^{8,43}$

The vast majority of patients were black $(96 \%)$ and unemployed (71.2\%), reflecting both the contribution of poor socio-economic circumstances to the risk of TB, and the differential utilisation of public hospitals along racial lines in our setting. According to the South African Census 2011, the drainage area of our facility consists of $76 \%$ black Africans; however, black African patients make up more than $90 \%$ of hospital inpatients. ${ }^{3}$ The majority of these patients live either in peri-urban informal settlements or underdeveloped urban living areas created during the period of racial segregation. The living conditions in these areas, characterised by overcrowding, have been demonstrated to promote the spread of tuberculosis. ${ }^{44,45}$

HIV co-infection was the leading risk factor (88.8\%) for EPTB in this study. Despite the widespread availability of ART in our 


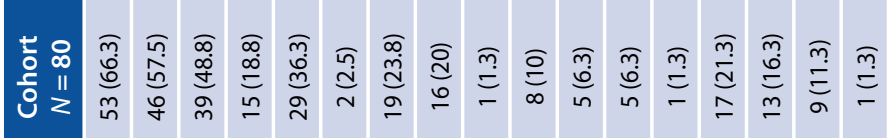

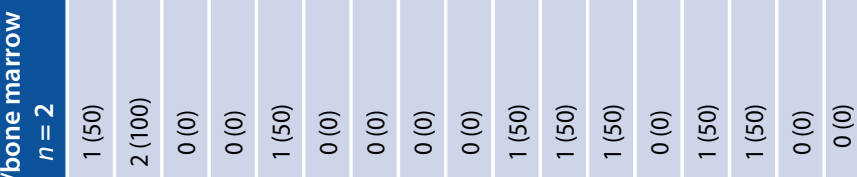

$\frac{0}{\infty}$

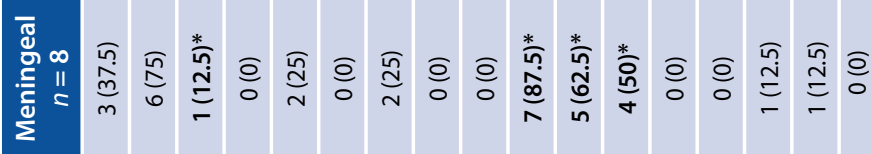

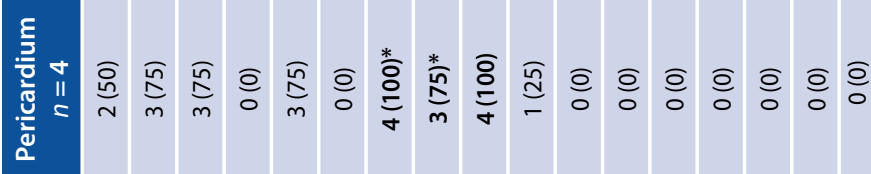

ธ

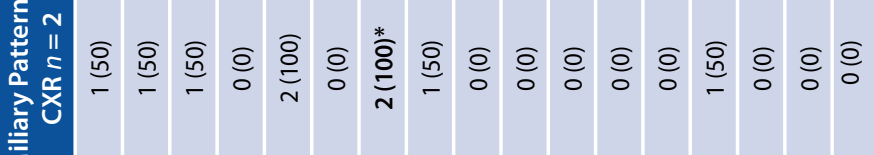

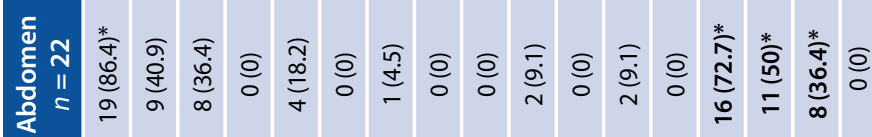

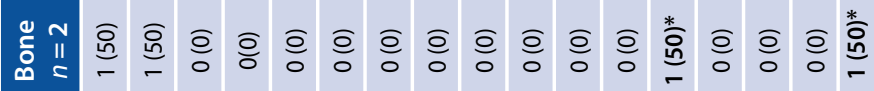

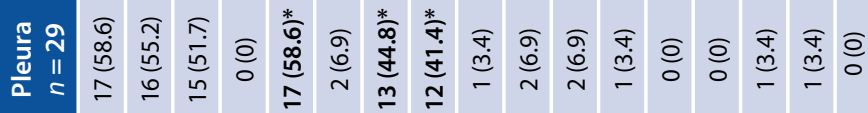

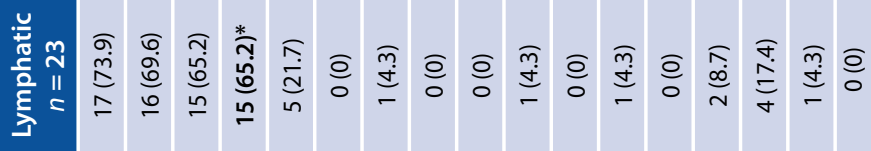

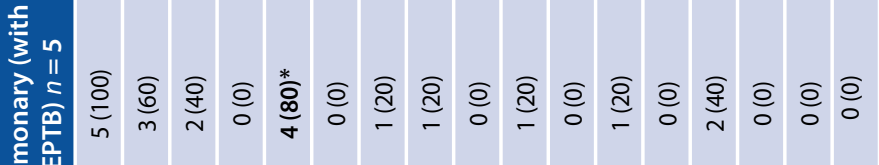

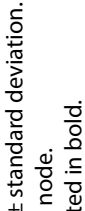

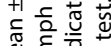

正

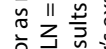

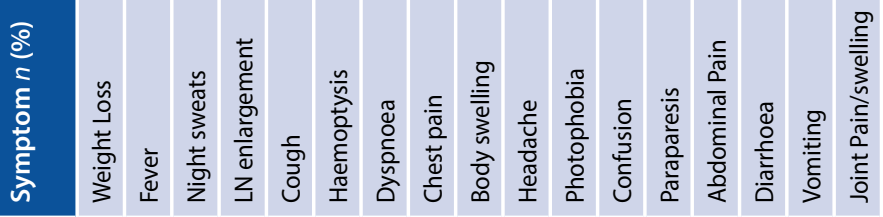

के 흥 흥흐 응 응응 
Table 4: Clinical signs according to site of tuberculosis infection

\begin{tabular}{|c|c|c|c|c|c|c|c|c|c|c|c|}
\hline $\begin{array}{l}\text { Sign } n \\
(\%)\end{array}$ & \multicolumn{2}{|c|}{$\begin{array}{l}\text { Pulmonary (with } \\
\text { EPTB) } n=5\end{array}$} & \multirow{2}{*}{$\begin{array}{l}\text { Lymphatic } \\
\qquad n=23 \\
12(52.2)\end{array}$} & \multirow{2}{*}{$\begin{array}{l}\text { Pleura } \\
n=29 \\
8(27.6)\end{array}$} & \multirow{2}{*}{ 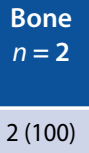 } & \multirow{2}{*}{$\begin{array}{c}\text { Abdomen } \\
n=22 \\
13(59.1)\end{array}$} & \multirow{2}{*}{$\begin{array}{c}\text { Miliary } \\
\text { Pattern on } \\
\text { CXR } n=2\end{array}$} & \multirow{2}{*}{$\begin{array}{l}\text { Pericardium } \\
\qquad \begin{array}{l}n=4 \\
4(100)\end{array}\end{array}$} & \multirow{2}{*}{$\begin{array}{l}\text { Meningeal } \\
\qquad \begin{array}{l}n=8 \\
5(62.5)\end{array}\end{array}$} & \multirow{2}{*}{$\begin{array}{c}\text { Blood/bone } \\
\text { Marrow } \\
n=2 \\
1(50)\end{array}$} & \multirow{2}{*}{$\begin{array}{l}\text { Cohort } \\
N=80\end{array}$} \\
\hline Tachyca & & $4(80)$ & & & & & & & & & \\
\hline Hypoter & sion & $4(80)^{*}$ & $2(8.7)$ & $0(0)$ & $2(100)$ & $3(13.6)$ & $1(50)$ & $1(25)$ & $1(12.5)$ & $1(50)$ & $8(10)$ \\
\hline Pyrexia & & $1(20)$ & $5(21.7)$ & $6(20.7)$ & $1(50)$ & $10(45.5)$ & $2(100)$ & $1(25)$ & $4(50)$ & $1(50)$ & 25 (31.3) \\
\hline Lympha & lenopathy & $2(40)$ & $23(100)^{*}$ & $5(17.2)$ & $0(0)$ & $8(36.4)$ & $1(50)$ & $1(25)$ & $2(25)$ & $0(0)$ & $33(41.3)$ \\
\hline Anaemi & & $2(40)$ & $5(21.7)$ & $11(37.9)$ & $0(0)$ & $5(22.7)$ & $1(50)$ & $4(100)$ & $5(62.5)$ & $1(50)$ & 25 (31.3) \\
\hline Clubbin & & $0(0)$ & $1(4.3)$ & $0(0)$ & $0(0)$ & $0(0)$ & $0(0)$ & $0(0)$ & $0(0)$ & $0(0)$ & $1(1.3)$ \\
\hline Anasarc & & $0(0)$ & $0(0)$ & $1(3.4)$ & $0(0)$ & $0(0)$ & $0(0)$ & $0(0)$ & $0(0)$ & $0(0)$ & $1(1.3)$ \\
\hline Mening & & $1(20)$ & $1(4.3)$ & $2(6.9)$ & $0(0)$ & $2(9.1)$ & $0(0)$ & $1(25)$ & $8(100)^{*}$ & $1(50)$ & $9(11.3)$ \\
\hline Consolic & ation & $1(20)$ & $1(4.3)$ & $1(3.4)$ & $0(0)$ & $2(9.1)$ & $0(0)$ & $0(0)$ & $1(12.5)$ & $1(50)$ & $5(6.3)$ \\
\hline Pleural & fusion & $0(0)$ & $1(4.3)$ & $28(96.6)^{*}$ & $0(0)$ & $1(4.5)$ & $0(0)$ & $1(25)$ & $3(37.5)$ & $1(50)$ & $29(36.3)$ \\
\hline Peritoni & & $0(0)$ & $0(0)$ & $0(0)$ & $0(0)$ & $6(27.3)^{*}$ & $0(0)$ & $0(0)$ & $0(0)$ & $0(0)$ & $6(7.5)$ \\
\hline Abdomi & al Mass & $1(20)$ & $0(0)$ & $0(0)$ & $0(0)$ & $1(4.5)$ & $0(0)$ & $0(0)$ & $0(0)$ & $0(0)$ & $1(1.3)$ \\
\hline Ascites & & $0(0)$ & $0(0)$ & $1(3.4)$ & $0(0)$ & $4(18.2)^{*}$ & $1(50)$ & $0(0)$ & $0(0)$ & $0(0)$ & $5(6.3)$ \\
\hline Hepator & egaly & $0(0)$ & $2(8.7)$ & $0(0)$ & $0(0)$ & $7(31.8)^{*}$ & $0(0)$ & $0(0)$ & $1(12.5)$ & $0(0)$ & $7(8.8)$ \\
\hline Gibbus & & $0(0)$ & $0(0)$ & $0(0)$ & $1(50)^{*}$ & $0(0)$ & $0(0)$ & $0(0)$ & $0(0)$ & $0(0)$ & $1(1.3)$ \\
\hline Arthritis & & $0(0)$ & $0(0)$ & $0(0)$ & $1(50)^{*}$ & $0(0)$ & $0(0)$ & $0(0)$ & $0(0)$ & $0(0)$ & $1(1.3)$ \\
\hline
\end{tabular}

Data expressed as $n$ (\%) or as mean \pm standard deviation.

$\mathrm{CXR}=$ chest radiograph.

Statistically significant results indicated in bold

*Indicates $p<0.05$. Fisher's exact test.

Table 5: Special investigations used to diagnose EPTB

\begin{tabular}{|l|c|}
\hline Method of diagnosis & $n(\%)$ \\
\hline Radiology* & $28(35)$ \\
\hline Microbiology** $^{*}$ & $52(65)$ \\
\hline Chemistry*** & $38(47.5)$ \\
\hline Microbiology plus chemistry & $32(40)$ \\
\hline Microbiology plus radiology & $17(21.25)$ \\
\hline Radiology plus chemistry & $4(5)$ \\
\hline
\end{tabular}

Data expressed as $n$ (\%), $n=$ number of tests conducted.

*Includes chest radiograph, computed tomography and ultrasound.

**Includes microscopy, culture and Xpert MDR/Rif.

***Includes fluid chemistry and adenosine deaminase levels.

health district, and the availability of HIV services at this facility, only $42.5 \%$ of patients with HIV were on ART. Importantly, all patients with HIV had a CD4 cell count below the threshold for initiation of ART at the time of the study. The mean CD4 cell count in patients with HIV was 68 cells $/ \mathrm{mm}^{3}$ overall and 106 cells $/ \mathrm{mm}^{3}$ (IQR $60-188$ ) in those patients on ART. This was in keeping with the well-established association between EPTB and low CD4 cell counts. As this study did not interrogate issues of adherence to ART, the degree of virological suppression or the duration of exposure to ART prior to this study, it is difficult to draw conclusions regarding the reason for the seemingly low CD4 cell counts in patients on ART. The presentation of patients with advanced untreated HIV is concerning in this setting, where the threshold for initiation of ART was $500 \mathrm{cell} / \mathrm{mm}^{3}$ at the time of the study. ${ }^{46}$ It remains uncertain whether the move to universal ART access together with a test-and-treat strategy will result in the anticipated reduction in the burden of TB. ${ }^{47,48}$

In keeping with epidemiological data from other settings, involvement of the lymph nodes (23\%), pleura (29\%) and abdomen $(22 \%)$ constituted the majority of extrapulmonary sites of disease. With the paucity of screening studies for EPTB and the absence of an adequate diagnostic tool, it is difficult to evaluate the true prevalence of the disease or accurately assess the anatomical distribution of disease. This diagnostic bias is unlikely to be resolved without the development of improved diagnostic tools. Tuberculosis involving the haematological system often requires an invasive diagnostic strategy including a bone marrow biopsy, which may not be easily available or accessible in resource-limited settings. The diagnosis of bone marrow involvement is often made presumptively in patients with haematological abnormalities in the presence of proven TB at other sites. Similarly for sites of TB involvement at other sites that require an invasive diagnostic strategy, there is often underreporting in favour of sites of involvement requiring a less invasive diagnostic strategy. The diagnosis of EPTB is often secured by identifying involvement of one extrapulmonary site, which favours the diagnosis of disease at sites that are most amenable to low-technology, minimally invasive diagnostic modalities. Only 15 patients (18.75\%) had the diagnosis of TB confirmed at more than one anatomical site in this study. A CD4 cell count of less than 100 cells $/ \mathrm{mm}^{3}$ demonstrated a significantly higher frequency of more than one site of EPTB. Karstaedt et al. performed a retrospective study of EPTB at a regional hospital in Johannesburg, South Africa in the pre-ART era, which revealed a similar predominance of pleural TB (39.1\%) and lymph node TB (31\%) among their patients. Significantly, in that study abdominal TB comprised only $2.9 \%$ of all cases of EPTB, as compared with $22 \%$ in our study. ${ }^{49}$ The inclusion of only culture-confirmed disease in that study systematically excluded sites of involvement such as abdominal TB, in which the diagnosis is often made using other diagnostic modalities. By including cases of EPTB based on the clinical diagnosis of EPTB, we provide a description of EPTB that more closely reflects our clinical experience with the disease. We recognise the inherent limitation of this approach in possibly including patients who were misdiagnosed with EPTB. This is a real concern in our setting where a retrospective study showed 
that 18 of 21 patients diagnosed with lymphoma over a fourmonth period were incorrectly diagnosed with TB in the preceding 12 months. ${ }^{50}$

Large retrospective series have been unable to make proper comparisons between features of EPTB because of the protean manifestations of the disease. In this study, weight loss (66.3\%), fever $(57.5 \%)$, night sweats $(48.8 \%)$ and cough $(29 \%)$ were the most common symptoms across all sites of disease. A study in Brazil found similar non-specific symptoms in their patients with EPTB. The differential diagnosis for the presence of these constitutional symptoms is broad, but remains an important signal for the diagnosis of TB in a high TB/HIV burden setting. While symptoms and signs are useful in directing further investigation to establish the site of disease, there is a paucity of data on discriminatory clinical features that may be used to support the diagnosis of TB over other pathologies at these sites.

A microbiologically confirmed diagnosis was made in only $65 \%$ of cases in this study. In the remaining cases, other laboratory data together with radiological studies were necessary to establish the diagnosis of EPTB. This reliance on laboratory and radiological data adds complexity to the diagnosis of the disease in resource-limited settings where such facilities are not widely available or accessible. It is important to note that $35 \%$ of patients were started on therapy without ever having a confirmed microbiological diagnosis. The outcome of these patients is not known because it lies outside the parameters of this study's objectives.

Use of Xpert MDR/Rif technology has improved the diagnosis of pulmonary TB and while its role in the diagnosis of EPTB is still being evaluated, the available data are promising. Pleural and cerebrospinal fluid specimens are consistently sent for Xpert MDR/Rif analysis at our facility. Xpert MDR/Rif testing of cerebrospinal fluid has been shown to be a useful rule-in test in other studies in our setting. Our experience with the use of Xpert MDR/Rif in pleural fluid analysis is similar to the available data, which suggest a limited role for the test in pleural fluid. In contrast, evaluation of pleural fluid ADA has emerged as a useful diagnostic study for suspected pleural involvement. In a high TB burden setting, pleural biopsy for suspected pleural TB has been largely obviated by the use of ADA. ${ }^{51}$

\section{Conclusion}

Extrapulmonary TB is a common disease entity in this high TB/ HIV burden setting. The clinical entity comprises a heterogeneous group of diseases described primarily by the anatomical site of involvement. In patients with a CD4 cell count of less than 100 cells $/ \mathrm{mm}^{3}$, clinicians should have a high index of suspicion of more than one organ system involvement. The clinical presentation of EPTB is protean, and establishing the diagnosis presents significant challenges in resource-limited settings. The current screening tools used in primary healthcare facilities and hospitals in South Africa screen for only pulmonary, lymphatic and pleural involvement. ${ }^{52} \mathrm{~A}$ new screening tool is needed in our setting given the high prevalence of abdominal TB and TB meningitis. The lack of diagnostic tools contributes to a diagnostic dilemma, and future developments in molecularbased technology may improve our ability to diagnose the disease appropriately. The use of available technologies requires greater evidence-based direction. Due to the reliance on nonmicrobiological diagnostic tests for EPTB, diagnostic certainty remains elusive. HIV continues to fuel the TB epidemic in this part of the world, and it remains to be seen whether the increased public health efforts in HIV will translate to a reduced burden of TB. Addressing the burden of EPTB will require our ongoing high index of suspicion and the use of innovative screening and diagnostic strategies.

Disclosure statement - No potential conflict of interest was reported by the authors.

\section{ORCID}

S Gounden (D) http://orcid.org/0000-0001-8892-2904

NP Magula (D) http://orcid.org/0000-0001-8625-9539

\section{References}

1. Mandell G, Bennet J, Dolin R. Principles and practice of infectious diseases. Philadelphia: Churchill Livingstone Elsevier; 2010.

2. Baddeley ADA, Dias HM, Falzon D. Floyd K Global tuberculosis report 2014. Geneva:WHO; 2014.

3. Lehohla P. Mortality and causes of death in South Africa 2010: findings from death notification. Pretoria: Fishwicks; 2013.

4. Kanabus A. TB statistics for South Africa-National \& provincial. UK: Global Health Education; 2016.

5. World Health Organization. Global tuberculosis Report 2016. Geneva: WHO; 2016.

6. Chakraborty MS. Tuberculosis and HIV illness. J Indian Med Assoc 2000;98:103-9.

7. Martinson NAH. Epidemiology of tuberculosis and HIV. Am Thorac Soc. 2011:288-93.

8. Sunnetcioglu A, Sunnetcioglu M, Binici I, et al. Comparative analysis of pulmonary and extrapulmonary tuberculosis of 411 cases. Ann Clin Microbiol Antimicrob. 2015;14:75. https://doi.org/10.1186/ s12941-015-0092-2

9. Statistic South Africa. Mid-year population statistics 2016. Pretoria: Stats SA; 2016.

10. Alvarez SMW. Extrapulmonary tuberculosis revisited: a review of experience at Boston City and other hospitals. Medicine. 1984;63:2555.

11. Bernardo J FvRC. Epidemiology and pathology of miliary and extrapulmonary tuberculosis. Wolters Kluwer; 2016.

12. Magee MJ, Foote M, Ray SM, et al. Diabetes mellitus and extrapulmonary tuberculosis: site distribution and risk of mortality. Epidemiol Infect. 2016;144:2209-16. https://doi.org/10.1017/ S0950268816000364

13. Hussey GLV. Miliary tubeculosis in children: a review of 94 cases. Pediatr Infect Dis J. 1991;10:832-6.

14. Perumal R, Padayatchi $N$, Naidoo $K$, et al. Understanding the profile of tuberculosis and human immunodeficiency virus coinfection: insights from expanded HIV surveillance at a tuberculosis facility in Durban, South Africa. ISRN AIDS. 2014;2014:260329.

15. Bernardo J FvRC. Clinical manifestations, diagnosis, and treatment of extrapulmonary and miliary tubeculosis. Wolters Kluwer; 2016.

16. Marjorie GP, Holenarasipur VR. Extrapulmonary tuberculosis: an overview. Am Fam Physician. 2005:1761-68.

17. Wilson D, Nachega J, Morroni C, et al. Diagnosing smear-negative tuberculosis using case definitions and treatment response in HIVinfected adults. The international journal of tuberculosis and lung disease : the official journal of the International Union against Tuberculosis and Lung Disease. 2006;10:31-8.

18. Chien HP, Yu MC, Wu MH, et al. Comparison of the BACTEC MGIT 960 with Lowenstein-Jensen medium for recovery of mycobacteria from clinical specimens. The international journal of tuberculosis and lung disease : the official journal of the International Union against Tuberculosis and Lung Disease. 2000;4:866-70.

19. Yuan M, Lyu Y, Chen ST, et al. Evaluation of Xpert MTB/RIF for the diagnosis of extrapulmonary tuberculosis in China. Biomed Environ Sci. 2016;29:599-602.

20. Tortoli ERC, Russo C. Clinical validation of Xpert MTB/RIF for the diagnosis of extrapulmonary tuberculosis. Eur Respir J. 2012;40:442-7. https://doi.org/10.1183/09031936.00176311

21. Naidoo P, Dunbar R, du Toit R, et al. Comparing laboratory costs of smear/culture and Xpert $^{\oplus}$ MTB/RIF-based tuberculosis diagnostic algorithms. Int J Tuberc Lung Dis. 2016;20:1377-85. 
22. Meyer-Rath G, Schnippel K, Long L, et al. The impact and cost of scaling up GeneXpert MTB/RIF in South Africa. PLoS One 2012;7:e36966. https://doi.org/10.1371/journal.pone.0036966

23. Lebina L, Abraham PM, Motlhaoleng K, et al. The use of decentralized GeneXpert by trained non-laboratory technicians in rural clinics in South Africa. Tuberculosis (Edinb). 2015;95:625-6. https://doi. org/10.1016/j.tube.2015.05.006

24. Cox HS, Daniels JF, Muller O, et al. Impact of decentralized care and the Xpert MTB/RIF test on rifampicin-resistant tuberculosis treatment initiation in Khayelitsha, South Africa. BMC Infect Dis. 2015;2:ofv014.

25. Hillemann D, Rusch-Gerdes S BC. Rapid molecular detection of extrapulmonary tuberculosis by the automated GeneXpert MTB/ RIF system. J. Clin. Microbiol. 2011;49:1202-1205. https://doi. org/10.1128/JCM.02268-10

26. Ghariani A, Jaouadi T, Smaoui S, et al. Diagnosis of lymph node tuberculosis using the GeneXpert MTB/RIF in Tunisia. Int J Mycobacteriol. 2015;4:270-5. https://doi.org/10.1016/j. ijmyco.2015.05.011

27. Tay TR, Tee A. Factors affecting pleural fluid adenosine deaminase level and the implication on the diagnosis of tuberculous pleural effusion: a retrospective cohort study. BMC Infect Dis. 2013;13:270. https://doi.org/10.1186/1471-2334-13-546

28. De Oliveira HG, Rossatto ER, Prolla JC. Pleural fluid adenosine deaminase and lymphocyte proportion: clinical usefulness in the diagnosis of tuberculosis. cytopathology. 1994;5:27-32. https://doi. org/10.1111/cyt.1994.5.issue-1

29. Salazar-Lezama M, Quiroz-Rosales H, Banales-Mendez JL, et al. Diagnostic methods of primary tuberculous pleural effusion in a region with high prevalence of tuberculosis. A study in Mexican population. Revista de investigacion clinica; organo del Hospital de Enfermedades de la Nutricion. 1997:49:453-6.

30. Salmanzadeh S, Tavakkol H, Bavieh K, et al. Diagnostic value of serum adenosine deaminase (ADA) level for pulmonary tuberculosis. Jundishapur J Microbiol. 2015;8:e21760.

31. Aggarwal AN, Agarwal R, Sehgal IS, et al Meta-analysis of Indian studies evaluating adenosine deaminase for diagnosing tuberculous pleural effusion. Int J Tuberc Lung Dis. 2016;20 (10):1386-91. https:// doi.org/10.5588/ijtld.16.0298

32. Diacon AH, Van de Wal BW, Wyser C, et al. Diagnostic tools in tuberculous pleurisy: a direct comparative study. Eur Respir J. 2003;22:589-91. https://doi.org/10.1183/09031936.03.00017103a

33. Ghosh GC, Sharma B, Gupta BB. CSF ADA determination in early diagnosis of tuberculous meningitis in HIV-infected patients. Scientifica (Cairo). 2016;2016:5820823.

34. Gui $X$, Xiao H. Diagnosis of tuberculosis pleurisy with adenosine deaminase (ADA): a systematic review and meta-analysis. Int J Clin Exp Med. 2014;7:3126-35.

35. Peter JG, Zijenah LS, Chanda D, et al. Effect on mortality of point-ofcare, urine-based lipoarabinomannan testing to guide tuberculosis treatment initiation in HIV-positive hospital inpatients: a pragmatic, parallel-group, multicountry, open-label, randomised controlled trial. Lancet. 2016;387:1187-97. https://doi.org/10.1016/S01406736(15)01092-2

36. Peter J, Theron G, Chanda D, et al. Test characteristics and potential impact of the urine LAM lateral flow assay in HIV-infected outpatients under investigation for TB and able to self-expectorate sputum for diagnostic testing. BMC Infect Dis. 2015;15:739. https://doi. org/10.1186/s12879-015-0967-z

37. Spalgais S, Jaiswal A, Puri M, et al. Clinical profile and diagnosis of extrapulmonary tb in HIV infected patients: routine abdominal ultrasonography increases detection of abdominal tuberculosis. Indian J Tuberc. 2013;60:147-53.
38. von Hahn T, Bange FC, Westhaus $\mathrm{S}$, et al. Ultrasound presentation of abdominal tuberculosis in a German tertiary care center. Scand J Gastroenterol. 2014;49:184-90. https://doi.org/10.3109/00365521.20 13.865258

39. Patel MN, Beningfield S, Burch V. Abdominal and pericardial ultrasound in suspected extrapulmonary or disseminated tuberculosis. S Afr Med J. 2011;101:39-42. https://doi.org/10.7196/SAMJ.4201

40. Perumal R, Padayatchi N, Stiefvater E. The whole is greater than the sum of the parts: recognising missed opportunities for an optimal response to the rapidly maturing TB-HIV co-epidemic in South Africa. BMC Public Health. 2009;9:938. https://doi.org/10.1186/1471-2458-9243

41. Kharsany $A B$, Frohlich JA, Yende-Zuma $N$, et al. Trends in HIV prevalence in pregnant women in rural South Africa. J Acquir Immune Defic Syndr. 2015;70: 289-95. https://doi.org/10.1097/ QAI.0000000000000761

42. Kharsany AB, Connolly $C$, Olowolagba $A$, et al. Increasing burden of pulmonary tuberculosis in young women. S Afr Med J. 2006;96:5245.

43. Gomes T, Reis-Santos B, Bertolde A, et al. Epidemiology of extrapulmonary tuberculosis in Brazil: a hierarchical model. BMC Infect Dis. 2014;14:119. https://doi.org/10.1186/1471-2334-14-9

44. Claassens $M$, van Schalkwyk C, den Haan $L$, et al. High prevalence of tuberculosis and insufficient case detection in two communities in the Western Cape. PLoS One. 2013;8:e58689. https://doi.org/10.1371/ journal.pone.0058689

45. David AM, Mercado SP, Becker D, et al. The prevention and control of HIV/AIDS, TB and Vector-borne diseases in informal settlements challenges, opportunities and insights. J Urban Health. 2007;84:6574. https://doi.org/10.1007/s11524-007-9183-5

46. National Department of Health. National consolidated guidelines for the prevention of mother-to-child transmission of HIV and the management of HIV in children, adholescents and adults. Pretoria: $\mathrm{NDOH} ; 2015$.

47. Suthar AB, Lawn SD, del Amo J, et al. Antiretroviral therapy for prevention of tuberculosis in adults with HIV: a systematic review and meta-analysis. PLoS Med. 2012;9:e1001270. https://doi.org/10.1371/ journal.pmed.1001270

48. Pretorius C, Menzies NA, Chindelevitch L, et al. The potential effects of changing HIV treatment policy on tuberculosis outcomes in South Africa: results from three tuberculosis-HIV transmission models. AIDS. 2014;28(Suppl 1):S25-34. https://doi.org/10.1097/ QAD.0000000000000085

49. Karstaedt AS. Extrapulmonary tuberculosis among adults: experience at Chris Hani Baragwanath Academic Hospital, Johannesburg. South Africa. S Afr Med J. 2014;104:22-4.

50. Puvaneswaran B, Shoba B. Misdiagnosis of tuberculosis in patients with lymphoma. S Afr Med J. 2012;103: 32-3. https://doi.org/10.7196/ SAMJ.6093

51. Valdes L, Alvarez D, San Jose $E$, et al. Value of adenosine deaminase in the diagnosis of tuberculous pleural effusions in young patients in a region of high prevalence of tuberculosis. Thorax. 1995;50:600-3. https://doi.org/10.1136/thx.50.6.600

52. National Department of Health. An assessment method and management tool for TB exposure at South African healthcare settings. Pretoria: NDOH. 2007.

Received: 11-03-2017 Accepted: 07-11-2017 